\title{
Eddy Structures Induced Within a Wedge by a Honing Circular Arc
}

C. P. Hills

Technological University Dublin, chris.hills@dit.ie

Follow this and additional works at: https://arrow.tudublin.ie/scschmatart

Part of the Applied Mathematics Commons, Applied Mechanics Commons, Fluid Dynamics Commons, and the Mathematics Commons

\section{Recommended Citation}

Hill, C.P.: Eddy Structures Induced Within a Wedge by a Honing Circular Arc, Theoretical and Computational Fluid Dynamics, Volume 15, Number 1 / September, 2001, pp.1-10. doi.org/10.21427/ cfw1-dn36

This Article is brought to you for free and open access by the School of Mathematics at ARROW@TU Dublin. It has been accepted for inclusion in Articles by an authorized administrator of ARROW@TU Dublin. For more information, please contact arrow.admin@tudublin.ie, aisling.coyne@tudublin.ie,gerard.connolly@tudublin.ie.

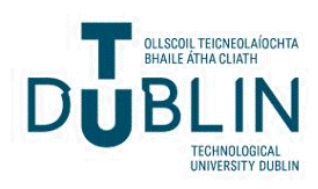




\title{
Eddy structures induced within a wedge by a honing circular arc
}

\author{
Christopher P. Hills \\ Lincoln College, Oxford, England OX1 3DR \\ e-mail: chris.hills@lincoln.ox.ac.uk $\quad$ tel: +441865288090
}

Submitted: February 5, 2001

\begin{abstract}
In this paper we outline an expeditious numerical procedure to calculate the Stokes flow in a corner due to the rotation of a scraping circular boundary. The method is also applicable to other wedge geometries. We employ a collocation technique utilising a basis of eddy (similarity) functions introduced by Moffatt (1964) that allows us to satisfy automatically the governing equations for the streamfunction and all the boundary conditions on the surface of the wedge. The circular honing problem thereby becomes one-dimensional requiring only the satisfaction of conditions on the circular boundary. The advantage of using the Moffatt eddy functions as a basis in wedge geometry is clear and the technique greatly reduces many of the concerns with accuracy and time expenditure associated with alternative numerical methods. An investigation of the details of the eddy structure for our particular geometry is presented.
\end{abstract}




\section{Introduction}

Recirculation regions, or eddies, are found throughout experimental and industrial fluid dynamics and commonly occur when viscous fluids encounter sharp corners (Moffatt (1964)) or surface cavities (Higdon (1985)). The importance of investigating the formation of eddies and closed paths in the complex streamline patterns that so frequently occur in applications cannot be over emphasised. Our understanding of eddy phenomena was significantly advanced by the study of Moffatt (1964) who analysed the two-dimensional Stokes flow in an infinite wedge of angle $2 \alpha$ due to an arbitrary (radially decaying) far-field disturbance. His study established that there is a critical half-angle $\left(\alpha_{\text {crit }}\right)$ for eddy formation and that a series solution can be constructed that is asymptotic in the radial distance from the wedge vertex. Underlying the analysis there is an eigenvalue problem that arises from the boundary conditions on the wedge walls which, for the case when $\alpha<\alpha_{\text {crit }}$ when recirculation regions will be present, possesses complex solutions. The associated eigenfunctions, here termed the Moffatt eddy functions and described more fully in Section 2, form the basis of a series solution.

Jeffrey \& Sherwood (1980) presented an analytic investigation of the formation of closed streamlines and eddies in several two-dimensional geometries. By studying stagnation points, corners and 'blocked' paths in simple Stokes flows, these authors extrapolate the streamlines of more complicated geometries. For example, they predict the flow patterns generated by a rotating cylinder placed in a shear flow and the streamlines for a shear flow past a cylinder touching a plane wall. Liu \& Joseph (1977) and more recently Khuri (1996) studied, by analytical means, the formation of eddies in a section of a wedge (corresponding to a polar region $\left[R_{1}, R_{2}\right] \times\left[\theta_{1}, \theta_{2}\right]$ ), but for driving mechanisms different to our own. These authors employ series expansions with a basis of (modified) Moffatt eddy functions and seek to determine unknown coefficients using biorthogonality relations. In the same vein, Hills (2000) also uses biorthogonality relations to demonstrate the presence of eddies (again arising from complex eigenvalues) for the low Reynolds number flow in a three-dimensional cylindrical geometry. The eddies, however, only arise in this geometry as a first order correction in the Reynolds number ${ }^{1}$. But, as a general rule, the analytic systems are rarely canonical and, to obtain a physical solution, it is usually necessary to resort to series truncation and the numerical evaluation of unknown coefficients.

In this paper we outline a general numerical method to determine the slow flow of a viscous fluid in a finite wedge geometry. We shall confine our attention to wedge angles $\alpha<\alpha_{\text {crit }}$ so that we may expect an eddy structure. To illustrate the method we consider a stationary wedge that is bounded by a circular arc whose centre, O, coincides with the wedge vertex. The slow flow is driven by the

\footnotetext{
${ }^{1}$ Liron \& Shahar (1978) and Blake (1979) have demonstrated that eddies can be induced for Stokes flow in the cylinder geometry by introducing delta function (Stokeslet) forcing.
} 
steady rotation of the curved boundary about an axis through O. The method is, however, flexible and easily adaptable to a variety of wedge-shaped Stokes flows: it will clearly cope with the flow within a triangle driven by the movement of one edge parallel to itself or the Stokes flow induced in a corner due to a tooth-shaped conveyor belt. Our aim is to outline a general method, ideally suited to wedge geometries, and, by investigating a particular case, obtain an insight into the eddy structure.

To resolve the Stokes flow we exploit the Moffatt eddy functions as a finite basis in a numerical collocation scheme. There are considerable advantages in using the eddy functions in a numerical solution over methods employing either finite difference or finite element approaches. First and foremost, the eddy functions all satisfy both the biharmonic equation and the boundary conditions on the wedge walls. Consequently the coefficients in the collocation scheme only have to ensure that the velocity boundary conditions are satisfied at discrete nodes on the rotating boundary: points within the remainder of the flow domain will then be automatically accommodated ${ }^{2}$. Thus, importantly, since it is known that the intensity of eddies decays exponentially towards the wedge vertex, our scheme avoids any difficulties associated with resolving small quantities near the vertex. In addition, collocation does not require lengthy calculation of grids and, unlike numerical adaptations of the biorthogonality method, will be quickly and easily adaptable to different wedge angles and geometries such as those described earlier.

The honing contact between wedge and circular boundary will clearly involve a discontinuity in fluid velocity. A similar situation occurs both in the classic Taylor paint scraper problem (Taylor (1962)) where two semi-infinite planes are held in scraping contact and in the three-dimensional extension of that problem, considered by Hills \& Moffatt (2000), in which an inclined plane is honed by a rotating lower plate. There is a question in all scraping problems of whether, physically, fluid leakage must occur at the point of velocity discontinuity on the boundaries. But, if the gap between the planes is smaller than the viscous penetration length, no fluid need escape. However the possibility of leakage and the introduction of sources and sinks to satisfy mass conservation leads to many interesting extensions of these problems. Consequences of a scraping (or honing) boundary condition are further discussed in Hills \& Moffatt (2000). The discontinuity in velocity will result in a Gibbs phenomenon at the point of contact between the stationary and moving boundaries but will not cause any significant numerical difficulties with the collocation scheme we set out in Section 3. Perhaps this is to be expected since locally, where the wedge meets the circular arc, we have effectively two perpendicular plates scraping

\footnotetext{
${ }^{2}$ It is found to be numerically advantageous to replace the boundary condition requiring the radial velocity to vanish on the circular arc by the easily obtained condition that the curved boundary is a streamline. By continuity, the streamfunction should have the same value as on the plates of the wedge.
} 


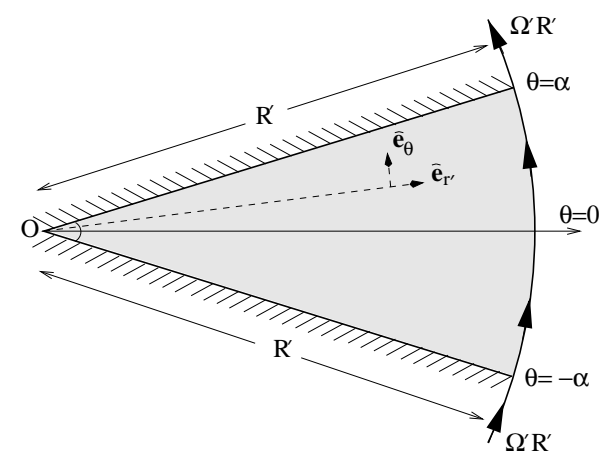

Figure 1: The geometry, parameters and coordinate system for the flow in a corner driven by a circular boundary.

past one another; a special case of the Taylor paint scraper. Thus the Stokes streamfunction, valid in the neighbourhood of this intersection, is known from the Taylor solution to be analytic. The discontinuity in the boundary condition is entirely reflected by a pole of order one in the pressure field.

We find that our method is extremely robust. The essential flow character is strongly dictated by the first few terms in the associated asymptotic sequence of Moffatt functions. Indeed, only a small number of basis functions is necessary to generate an accurate solution and to reveal the eddy structure. In particular, we determine as a function of the internal wedge angle the penetration of the induced flow into the corner and suggest a plausible method of eliminating in practical applications eddies and insular recirculation regions.

\section{Problem formulation and basis functions background}

We consider an incompressible, linearly viscous fluid, of kinematic viscosity $\nu^{\prime}$, contained in the region formed by a finite wedge of side $R^{\prime}$ and internal angle $2 \alpha$, that is in contact with a circular arc, radius $R^{\prime}$ with centre, $O$, at the vertex of the wedge. The fluid is maintained in two-dimensional steady motion, with velocity field $\mathbf{u}^{\prime}$, by the rotation of the curved boundary with constant angular speed $\Omega^{\prime}$ about an axis through $O$, perpendicular to the plane of the flow. We employ a plane polar coordinate system $\left(r^{\prime}, \theta\right)$, origin at $O$, in which the corner is symmetrically placed about the horizontal $(\theta=0)$ (see Figure 1). The flow within the fluid is assumed so slow that the Stokes equations apply and the Reynolds number, $R e=\Omega R^{\prime 2} / \nu^{\prime} \ll 1$. Consequently, our analysis will apply to wedges of small radii, small angular speeds or to very viscous fluids. It is convenient to work in terms of non-dimensional variables $r, \mathbf{u}$ defined in terms of the characteristic length $R^{\prime}$ and speed $\Omega^{\prime} R^{\prime}$ by

$$
r=r^{\prime} / R^{\prime} \quad \mathbf{u}=\mathbf{u}^{\prime} / \Omega^{\prime} R^{\prime}
$$


Moreover, the incompressibility of the fluid and the two-dimensional nature of the flow allows us to formulate the governing equations in terms of a dimensionless streamfunction, $\psi(r, \theta)$, as

$$
\begin{aligned}
\mathbf{u} & =-r^{-1} \partial \psi / \partial \theta \widehat{\mathbf{e}}_{r}+\partial \psi / \partial r \widehat{\mathbf{e}}_{\theta} \\
\nabla^{4} \psi & =0
\end{aligned}
$$

where $\nabla^{2}$ denotes the standard second order, two-dimensional Laplacian operator in the $(r, \theta)$-variables and $\widehat{\mathbf{e}}_{r}, \widehat{\mathbf{e}}_{\theta}$ are the usual polar base vectors.

The velocity boundary conditions on the wedge and the circular boundary require ${ }^{3}$

$$
\begin{aligned}
& \frac{\partial \psi}{\partial r}=\frac{\partial \psi}{\partial \theta}=0 \quad \text { on } \quad \theta= \pm \alpha, \\
& \frac{\partial \psi}{\partial r}=1, \quad \frac{\partial \psi}{\partial \theta}=0 \quad \text { on } \quad r=1,
\end{aligned}
$$

and it is immediately clear that there is a discontinuity in the azimuthal component of the velocity $(\partial \psi / \partial r)$ at the point of contact $(r=1, \theta= \pm \alpha)$. As we have previously mentioned, such discontinuities are not unknown (see Hills \& Moffatt (2000) for a fuller discussion). Locally the wedge and circular boundary contact can be modelled as two scraping perpendicular planes. Taylor (1962) showed that in such a configuration the velocity discontinuity at the contact leads to an analytic streamfunction but, at the contact point, the pressure has a singularity of order one.

It is well known that in plane polar coordinates the biharmonic equation $(2.2 \mathrm{~b})$ possesses, in the terminology of Barenblatt (1996), similarity solutions of the second kind with a streamfunction, $\psi$, having the form $r^{\lambda} f_{\lambda}(\theta)$. The particular cases when $\lambda=0,1,2$ respectively correspond to a source at the origin, a linearly scraping plate (Taylor (1962)) and a closing hinge. Moffatt (1964) considered a sub-class of these similarity solutions that also satisfy homogeneous boundary conditions on the walls of the infinite wedge. We shall limit our discussion to the case of a symmetrically placed wedge when (2.3a) applies. The streamfunction will then be even in $\theta$ and the boundary condition leads to the eigenvalue equation (see Moffatt (1964), eq. (3.5))

$$
\sin 2(\lambda-1) \alpha=-(\lambda-1) \sin 2 \alpha
$$

for the determination of the parameter $\lambda$. The solutions of (2.4) depend crucially upon the wedge geometry and Moffatt showed that there exists a critical half-angle, $\alpha_{\text {crit }}$. For wedge half-angles $\alpha>$ $\alpha_{\text {crit }}$ the values of $\lambda$ are real and eddies do not appear but, for $\alpha<\alpha_{\text {crit }}$ the eigenvalues are complex and lead to the complex streamfunction $\Psi$ (see Moffatt (1964), eq. (3.9)),

$$
\Psi=\mathcal{A} r^{\lambda}\left(\frac{\cos \lambda \theta}{\cos \lambda \alpha}-\frac{\cos (\lambda-2) \theta}{\cos (\lambda-2) \alpha}\right) \text {. }
$$

\footnotetext{
${ }^{3}$ Note that the problem is now entirely formulated in terms of non-dimensional variables and the flow pattern will be solely dependent upon $\alpha$. Only the intensity and size of the flow are given by the physical parameters.
} 
The (even) similarity solution that we are concerned with has $\alpha_{\text {crit }} \simeq 73^{\circ}$. We can use the linearity of the Stokes equation to construct the physical solution $\psi$ as the real part of $(2.5)$, that is $\psi=\mathbb{R}(\Psi)$. The nature of the physical solution is immediately deducible from (2.5). In particular, if we approach the vertex of the wedge along a ray $(\theta=$ constant), we see that when $\lambda$ is complex, the sign of the streamfunction alternates. Evidently there is an infinite cascade of recirculation regions whose intensity decays exponentially as the vertex is approached. We shall concentrate on wedge angles $\alpha<\alpha_{\text {crit }}$, for which the eigenvalues are complex and eddies do appear.

It is convenient to order the sequence $\left\{\lambda_{k}\right\}$ by increasing real part so that

$$
1<\mathbb{R} \lambda_{1}<\mathbb{R} \lambda_{2}<\mathbb{R} \lambda_{3}<\cdots
$$

Moffatt \& Duffy (1980) have illustrated the dependence of these eigenvalues on the half-angle $\alpha$ (see Moffatt \& Duffy (1980), Figure 7). We note that $\mathbb{R} \lambda_{k}>1$ so that the velocity given by (2.2a) is necessarily finite as we approach the vertex. In fact, Moffatt $(1964)$ showed that $1+(2 k-1) \pi / 2 \alpha<$ $\mathbb{R} \lambda_{k}<1+(2 k-1 / 2) \pi / 2 \alpha$. Again, using the linearity of (2.2)-(2.3), we can construct a physical (real-valued) streamfunction satisfying $(2.2 \mathrm{~b})$ and $(2.3 \mathrm{a})$ as

$$
\psi=\mathbb{R}\left\{\sum_{k=1}^{N} \mathcal{A}_{k} r^{\lambda_{k}}\left(\frac{\cos \lambda_{k} \theta}{\cos \lambda_{k} \alpha}-\frac{\cos \left(\lambda_{k}-2\right) \theta}{\cos \left(\lambda_{k}-2\right) \alpha}\right)\right\} .
$$

where the $\mathcal{A}_{k}$ are arbitrary complex constants and $N$ can take any positive integer value (the general solution corresponding to $N \rightarrow \infty)$. The eigenvalue ordering (2.6) we have imposed ensures that $(2.7)$ is asymptotic in $r$ as we approach the wedge vertex.

The general solution (2.7) forms the foundation of our method of solving the circularly-bounded wedge problem (2.2b)-(2.3b). Essentially we recognise that the Moffatt eddy functions, ordered as above, are an ideal basis for our wedge geometry. Not only do they automatically satisfy the conditions on all but one boundary, but the contributions from each term in the basis is exponentially decreasing as we approach the corner. The principal contribution stems from the basis function associated with $\lambda_{1}$. In the next section, we shall use a truncated series and will determine the coefficients $\mathcal{A}_{k}$ by numerical collocation. The eddy functions we use need not form a complete set for all permissible wedge flows but we obtain a physically realistic solution approximating all dynamic requirements from the set spanned by these eddy functions. In order to judge the accuracy of our approximate solution we need only monitor how closely the solution matches the boundary conditions on the circular arc. 


\section{The numerical scheme and results}

Our collocation scheme for determining the physical streamfunction $\psi$ uses for a given value of $N$ a series of the form (2.7). The coefficients $\mathcal{A}_{k}(k=1,2, \ldots N)$ in $(2.7)$ are complex and will each require two constraints to resolve our series approximation to the wedge flow. The constraints are obtained by satisfying the conditions on $\psi$ at discrete (collocation) points on $r=1$. Formally, at any point on the arc we have two boundary conditions and so will need a total of $N$ collocation points where the required components of velocity are strictly enforced. We shall choose these points in the positive range $^{4} \theta \in[0, \alpha)$. The finite series that constitutes our collocation function is, by construction, even in $\theta$ so that we will tacitly satisfy the boundary conditions at either $2 N-1$ (if $\theta=0$ is a collocation point) or $2 N$ boundary points over the whole arc. A measure of the effectiveness of our scheme will be the number, $N$, of basis functions we require in the series in order to obtain a physically satisfactory solution everywhere.

Let us briefly consider the positioning of the collocation points. For simplicity the points will be equally spaced. The intrinsic symmetry of the finite series with respect to $\theta$ means that $\partial \psi / \partial \theta$ will always vanish at $\theta=0$. Thus, although we may use points on the boundary corresponding to

$$
r=1, \quad \theta=k \alpha / N, \quad k=0,1,2, \ldots, N-1
$$

for the boundary condition on $\partial \psi / \partial r$, were we to use these points also for $\partial \psi / \partial \theta$ we would be lacking one condition. A possible method to overcome this shortfall would be to employ the collocation set

$$
r=1, \quad \theta=k \alpha /(N+1), \quad k=1,2, \ldots, N
$$

But there is another approach. The circular boundary is a streamline. In fact, by continuity, the streamfunction has the same value on the arc as on the wedge walls and we may replace the condition on $\partial \psi / \partial \theta$ given in $(2.3 \mathrm{~b})$ by explicitly specifying $\psi$. Our alternative set of boundary conditions can therefore be written as

$$
\psi(1, \theta)=0 \quad \text { and } \quad \partial \psi / \partial r(1, \theta)=1
$$

Numerical comparison of schemes which employed the set (3.2) for the determination of either $i$ ) both derivatives $\partial \psi / \partial r, \partial \psi / \partial \theta$ or $i i) \partial \psi / \partial \theta$ alone (with $\partial \psi / \partial r$ obtained using (3.1)), with our alternative boundary conditions applied at (3.1) showed that (3.3) was the most effective way to determine coefficients. Far fewer basis functions are needed to obtain an accurate solution under this method and we shall therefore, henceforth, adopt the boundary conditions (3.3) on $\psi$ and $\partial \psi / \partial r$ at the collocation

\footnotetext{
${ }^{4}$ The cases $\theta= \pm \alpha$ must be excluded due to the discontinuity in fluid velocity at these points.
} 
points of (3.1). There is a heuristic argument to support the use of this alternative boundary condition. The condition $\partial \psi / \partial \theta=0$ attempts to make $\psi$ constant without explicit knowledge of what that constant should be: the constant will follow from numerical continuity. However, adopting the alternative form of the boundary condition we explicitly specify the constant $(\psi=0)$. Exploiting the symmetry and the fact that the streamfunction vanishes on the wedge walls, we have that $\psi=0$ at $2 N+1$ points on the arc corresponding to $\theta=k \alpha / N$ with $k=-N, \ldots, N$. Thus, from Rolle's theorem, the streamfunction will necessarily have $N$ turning points on the upper half-arc where $\partial \psi / \partial \theta=0$.

For a given half-corner angle, $\alpha$, we determine the first $N$ eigenvalues $\lambda_{1}, \ldots, \lambda_{N}$ satisfying (2.4). We then determine the complex constants $\mathcal{A}_{k}$ from the $2 N$ simultaneous linear equations resulting from the boundary conditions of (3.3) at the collocation points (3.1). Of course, in general, the streamfunction will not vanish at points in between the collocation points and this will physically correspond to a mass flux across the boundary. We monitor, therefore, the accuracy of our solutions by a plot of $\psi(1, \theta)$. In a

plot of streamlines in the region $(r \leq 1, \theta \in[-\alpha, \alpha])$ a non-vanishing $\psi$ on $r=1$ will manifest itself by broken streamlines at this boundary. It is worth reiterating that our solution automatically satisfies all physical requirements in the remainder of the wedge and it is sufficient to ensure an adequate treatment of the circular boundary.

We now set out the results for two representative wedges with half-wedge angles $\alpha=15^{\circ}$ and $\alpha=30^{\circ}$. In both cases as few as twenty terms $(N=20)$ yields an accurate, physical solution but for purposes of comparison and understanding the structure of the composite eddy picture we include some intermediate cases.

\subsection{Half-wedge angle $\alpha=15^{\circ}$}

We list in Table 1 the real and imaginary parts of the first twenty eigenvalues from equation (2.4) for $\alpha=15^{\circ}$ and in Table 2 we set out the series coefficients of $(2.7)$ for the four cases $N=1,5,10,20$. The coefficients are determined from the set of simultaneous equations arrived at by applying the boundary conditions of (3.3) at the collocation points. We observe that the real parts of the $\lambda_{k}$ increase rapidlywith $k$, in accordance with the bound given by Moffatt (1964). Therefore we can see that as we approach the vertex of the corner $(r \ll 1)$ the series $(2.7)$ will very quickly become dominated by the initial term of the series. A second observation concerns the constants $\mathcal{A}_{k}$. Their values appear remarkably constant as we increase the number of terms $N$ in the series. This is not a universal feature of the method of collocation (coefficients can be volatile) but it seems to suggest that the initial terms of the series are also essentially dominant on $r=1$; the later terms act as corrections.

In Figure 2 we show for the cases $N=1,5$ and 20 the streamlines inside the wedge. In each case 


\begin{tabular}{|c|c|c||c|c|c||c|c|c||c|c|c|}
\hline \multicolumn{10}{|c|}{ Ordered Eigenvalues of equation $(2.4): \lambda_{k}=p_{k}+\mathrm{i} q_{k}$} \\
\hline \hline$k$ & $p_{k}$ & $q_{k}$ & $k$ & $p_{k}$ & $q_{k}$ & $k$ & $p_{k}$ & $q_{k}$ & $k$ & $p_{k}$ & $q_{k}$ \\
\hline 1 & 9.063 & 4.203 & 6 & 69.776 & 8.093 & 11 & 129.863 & 9.284 & 16 & 189.899 & 10.013 \\
2 & 21.467 & 5.837 & 7 & 81.802 & 8.398 & 12 & 141.872 & 9.454 & 17 & 201.904 & 10.130 \\
3 & 33.613 & 6.693 & 8 & 93.822 & 8.661 & 13 & 153.880 & 9.610 & 18 & 213.908 & 10.241 \\
4 & 45.691 & 7.281 & 9 & 105.838 & 8.892 & 14 & 165.887 & 9.754 & 19 & 225.912 & 10.345 \\
5 & 57.741 & 7.730 & 10 & 117.852 & 9.098 & 15 & 177.893 & 9.887 & 20 & 237.916 & 10.444 \\
\hline
\end{tabular}

Table 1: The first twenty ordered eigenvalues of equation (2.4) relating to a wedge with half-angle $\alpha=15^{\circ}$

\begin{tabular}{|lcccc|}
\hline $\mathbf{N}=\mathbf{1}$ & & & & \\
$(0.4327,0.4144)$ & & & & \\
$\mathbf{N}=\mathbf{5}$ & & & & \\
$(0.5628,0.5369)$ & $(0.2456,0.2607)$ & $(0.1405,0.1911)$ & $(0.0532,0.1396)$ & $(-0.0196,0.3390)$ \\
$\mathbf{N}=\mathbf{1 0}$ & & & & \\
$(0.5613,0.5349)$ & $(0.2436,0.2507)$ & $(0.1521,0.1719)$ & $(0.1099,0.1344)$ & $(0.0840,0.1140)$ \\
$(0.0630,0.1021)$ & $(0.0400,0.0933)$ & $(0.0099,0.0788)$ & $(-0.0216,0.0407)$ & $(-0.0204,0.0039)$ \\
$\mathbf{N}=\mathbf{2 0}$ & & & & \\
$(0.5610,0.5347)$ & $(0.2419,0.2495)$ & $(0.1496,0.1686)$ & $(0.1076,0.1281)$ & $(0.0840,0.1038)$ \\
$(0.0691,0.0878)$ & $(0.0587,0.0767)$ & $(0.0510,0.0687)$ & $(0.0449,0.0629)$ & $(0.0396,0.0586)$ \\
$(0.0347,0.0556)$ & $(0.0297,0.0534)$ & $(0.0243,0.0517)$ & $(0.0178,0.0502)$ & $(0.0098,0.0483)$ \\
$(-0.0004,0.0445)$ & $(-0.0126,0.0364)$ & $(-0.0240,0.0202)$ & $(-0.0233,-0.0032)$ & $(-0.0055,-0.0047)$ \\
\hline
\end{tabular}

Table 2: The complex pairs $\left(a_{k}, b_{k}\right)$ of $\mathcal{A}_{k}\left(=a_{k}+\mathrm{i} b_{k}\right)$ for a wedge of half-angle $\alpha=15^{\circ}$. The coefficients $\mathcal{A}_{k}, k=1,2, \ldots, N$, are to be read horizontally.

contours were chosen which intersected the axis $\theta=0$ at even intervals in order to describe the whole geometry. However, since each contour cuts the axis of symmetry twice their second intersection cannot be governed and so the overall appearance is not uniform. But the plots do not give a measure of flow intensity. The eddy cells, described by Moffatt (1964), are clear and, interestingly, the sizes vary very little between plots. The main difference between the three plots that make up Figure 2 occurs in the vicinity of the boundary $r=1$. The top plot has numerous broken streamlines (indicative of a mass flux). However, for $N=20$, there are no broken streamlines and the solution is physically acceptable. For this value of $N$ the eddies are centred on the symmetry axis at $r=0.2,0.4,0.9$, with the respective values of $\psi$ being $^{5}-0.7 \times 10^{-7}, 0.6 \times 10^{-4},-0.047$. The intensity, therefore, decreases as we approach the corner with the sense of circulation in adjacent cells alternating.

\footnotetext{
${ }^{5}$ These intensities are in agreement with the magnitudes found in studies of eddies in similar geometries such as those by Moffatt (1964) and Liu \& Joseph (1977).
} 
a)

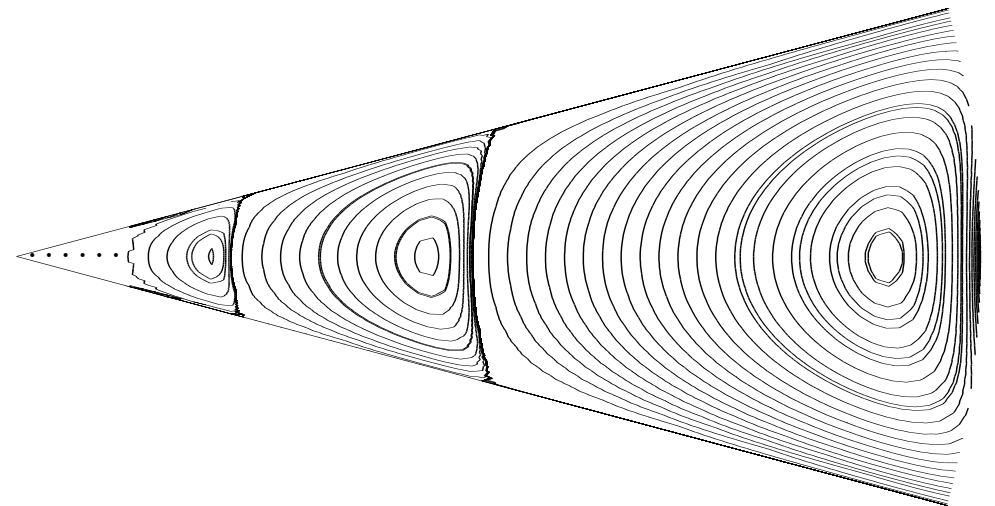

c)

b)
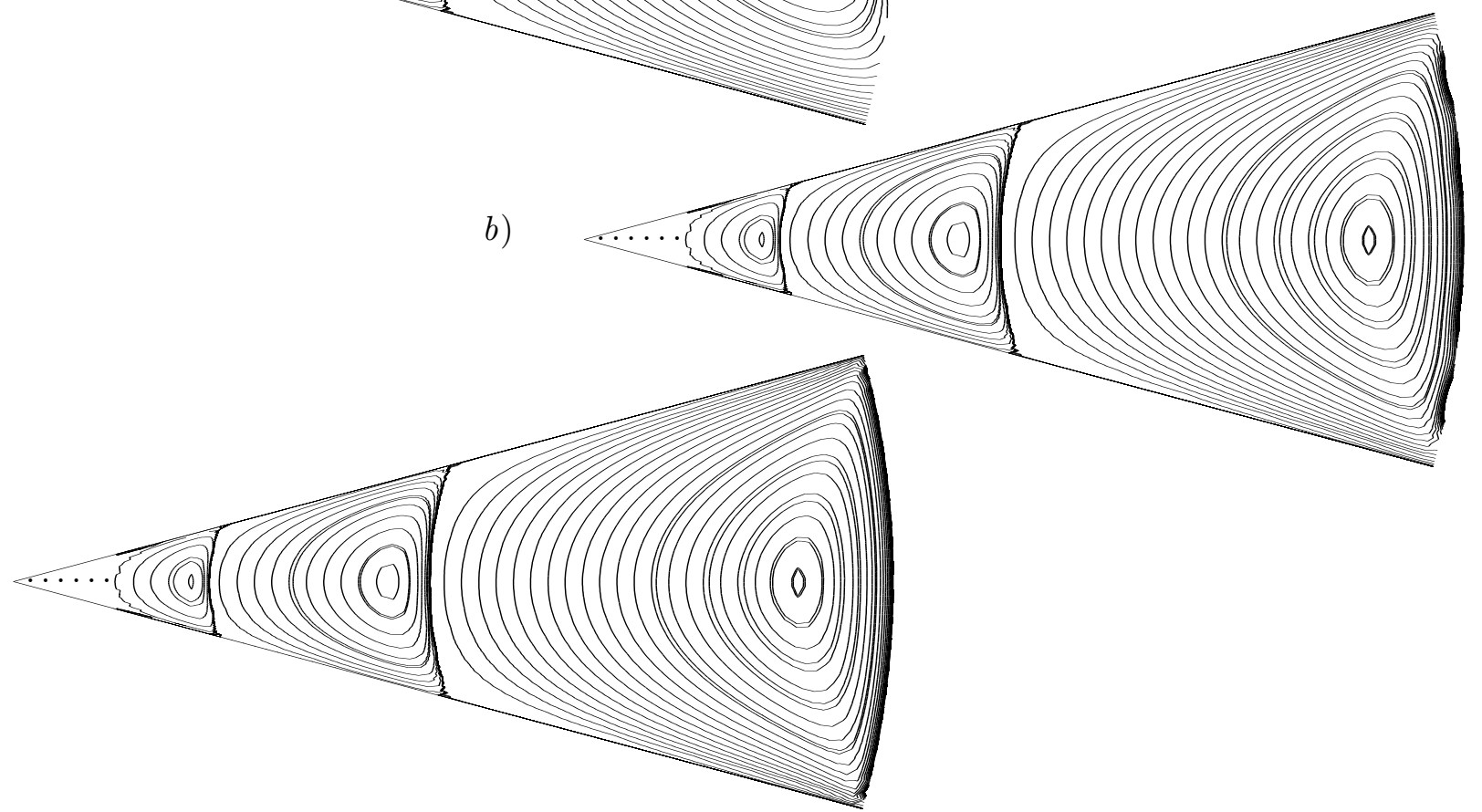

Figure 2: Streamline plots of the principal eddies in an infinite sequence that decrease in size towards the wedge vertex. We plot the finite series (2.7) for a corner of half-angle $\alpha=15^{\circ}$ in the cases $a$ ) $N=1, b) N=5, c) N=20$. The case $N=1$ demonstrates the dominance of the first term of the series but the broken streamlines show that the boundary condition is not completely satisfied.

As a further check on the solutions, we display in Figure 3 the streamfunction, $\psi$, on the boundary $r=1$ across the full range ${ }^{6} \theta \in[-\alpha, \alpha]$. Case $(c)$ confirms that, to within numerical error, $\psi$ substantially vanishes on the arc $r=1$. The only places where we have any significant deviation are at the extremities $\theta= \pm \alpha$ where there is clearly a manifestation of the Gibbs phenomenon.

Qualitatively, at the resolution of Figure 2, the flow substantially consists of three cells of rapidly decreasing dimension. The streamline $\psi=0$ is a streamline of separation, marking the boundary between adjacent cells and is the only streamline to intersect the wedge boundary. The streamfunction is of opposite signs in adjacent cells (recall $\psi$ changes sign infinitely often as we approach the vertex) so that the circulation in adjacent cells has opposite sense. A characteristic feature of the eddy structure

\footnotetext{
${ }^{6}$ The scale in Figures $3(a)-(c)$ is not uniform.
} 


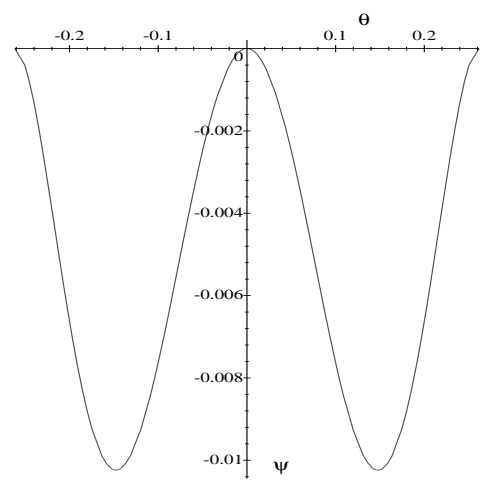

(a)

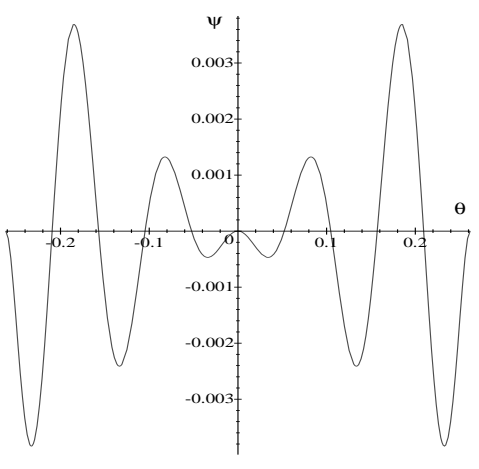

(b)

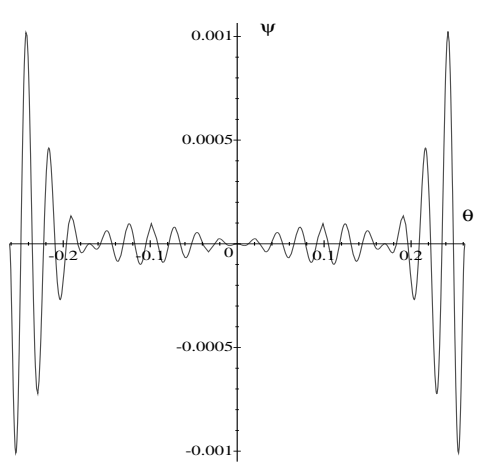

$(c)$

Figure 3: Plot of the finite series (2.7) evaluated on $r=1$ for the cases $a$ ) $N=1, b) N=5, c) N=20$. The deviation in $(c)$ near $\alpha=15^{\circ}$ is due to the discontinuity in velocity at the honing contact.

\begin{tabular}{|c|c|c||c|c|c||c|c|c||c|c|c|}
\hline \multicolumn{10}{|c|}{ Ordered Eigenvalues of $(2.4): \lambda_{k}=p_{k}+\mathrm{i} q_{k}$} \\
\hline \hline$k$ & $p_{k}$ & $q_{k}$ & $k$ & $p_{k}$ & $q_{k}$ & $k$ & $p_{k}$ & $q_{k}$ & $k$ & $p_{k}$ & $q_{k}$ \\
\hline 1 & 5.059 & 1.952 & 6 & 35.392 & 3.909 & 11 & 65.433 & 4.505 & 16 & 95.451 & 4.869 \\
2 & 11.246 & 2.778 & 7 & 41.404 & 4.061 & 12 & 71.438 & 4.589 & 17 & 101.453 & 4.928 \\
3 & 17.314 & 3.208 & 8 & 47.414 & 4.193 & 13 & 77.442 & 4.667 & 18 & 107.455 & 4.983 \\
4 & 23.351 & 3.502 & 9 & 53.422 & 4.309 & 14 & 83.445 & 4.739 & 19 & 113.457 & 5.035 \\
5 & 29.375 & 3.727 & 10 & 59.428 & 4.412 & 15 & 89.448 & 4.806 & 20 & 119.459 & 5.085 \\
\hline
\end{tabular}

Table 3: The first twenty ordered eigenvalues of (2.4) relating to half-corner angle $\alpha=30^{\circ}$

is that the fluid in each cell may never travel into an adjacent cell and is trapped within. Therefore the proportion of the fluid nearest the vertex beyond the principal cell is an interesting property and is discussed more fully in the final subsection.

\subsection{Half-wedge angle $\alpha=30^{\circ}$}

To understand the influence of wedge angle size we consider the corner angle $\alpha=30^{\circ}$. The eigenvalues of (2.4) are listed in Table 3. As before, the real part of $\lambda_{k}$ increases rapidly so that the later terms of the series will again quickly become dominated by the first as we approach the corner: the contribution of these terms will be most influential near $r=1$. Again an accurate physical solution is obtained for $N=20$. In Figure 4 we plot streamlines of the wider angled wedge. Substantially the picture is the same: there are characteristic alternating eddies separated by streamlines $\psi=0$ which intersect the wedge boundary wall. But here the principal eddy is much more dominant. The centres of the two largest eddies are now approximately at positions $r=0.16$ and 0.8 and the proportion of fluid 


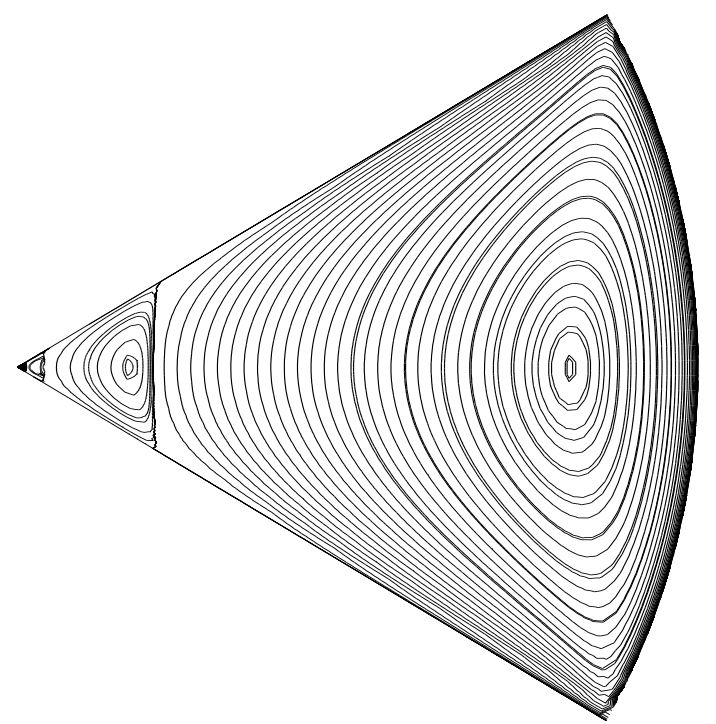

Figure 4: Streamline plot for the flow in a corner of half-angle $\alpha=30^{\circ}$ for the finite series (2.7) with $N=20$.

outside the principal eddy is reduced. In other words, the flow penetrates the corner to a greater extent supporting the intuitive notion that the wider the geometry the further the external flow can enter.

\subsection{Eddy penetration vs. half-wedge angle $\alpha$}

Let us return to a general wedge angle $2 \alpha$ and quantify the extent to which the flow pattern penetrates the corner. An obvious measure of this feature is the position $\left(r=d_{1}\right)$ on the axis of symmetry of the intersection of the dividing streamline nearest the circular boundary. We also measure the second intersection $\left(r=d_{2}\right)$ of the dividing streamline but any closer to the vertex the flow is extremely weak. Figure 5 shows the variation of the distances $d_{1}$ and $d_{2}$ with angle $2 \alpha\left(0 \leq \alpha \leq \alpha_{\text {crit }}\right)$. In the limit $\alpha \rightarrow 0$ both $d_{1}, d_{2}$ tend to one: both distances are non-dimensionalised and the flow cannot enter the corner $^{7}$. As the wedge angle increases the distances $d_{1}, d_{2}$, decrease rapidly: for $\alpha=15^{\circ}, d_{1}=0.45$, $d_{2}=0.22$ and for $\alpha=30^{\circ}, d_{1}=0.2, d_{2}=0.04$. The limit of both $d_{1}$ and $d_{2}$ must be zero as $\alpha$ approaches $\alpha_{\text {crit }}$.

The level of flow penetration into a corner is likely to be an important concern in the design of physical applications and, from Figure 5, the confining effects of eddies on the fluid can be countered by making angles as wide as possible. Clearly, if eddies are to be avoided completely, one method is to ensure that any corner angles are always greater than the critical angle, $\alpha_{\text {crit }}$, determined by

\footnotetext{
${ }^{7}$ We may also think of the limit $\alpha \rightarrow 0$ as representing the flow between two infinite parallel plates. An infinite sequence of eddies is formed, each of finite diameter, whose size in proportion to the 'corner' is negligible.
} 


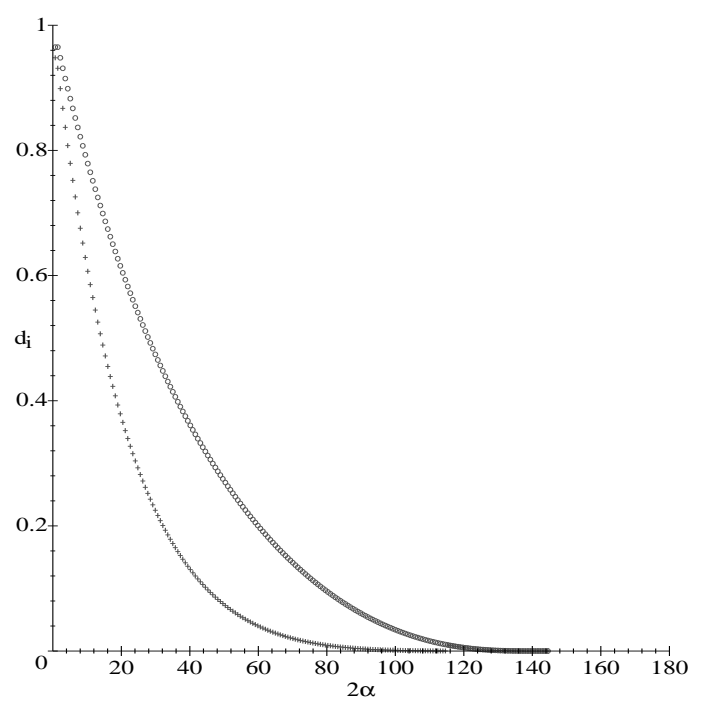

Figure 5: Plot showing the distance from the vertex of the first and second intersections of the streamline $\psi=0$ with the axis $\theta=0, d_{1}$ and $d_{2}$ respectively, against total wedge angle $2 \alpha$.

Moffatt (1964): there are no eddies for $\alpha>\alpha_{\text {crit }}$. But, this is not always a practical solution and there is an alternative approach that is suggested by the contour plots of Figures 2 and 4 . If the geometry of a corner is modified so that its boundaries now coincide with the dividing streamline between the largest and second largest eddy, the Stokes flow in the new corner will take the form of a single circulation (unchanged from the original streamfunction) and the main flow will penetrate fully to all the boundaries.

\section{Acknowledgements}

I am very grateful to my thesis advisor, Professor H.K. Moffatt FRS, for his encouragement and many helpful contributions. My research was supported by EPSRC, U.K.

\section{References}

Barenblatt, G. I. 1996 Scaling, Self-similarity, and Intermediate Asymptotics. Cambridge University Press

BLAKE J. 1979 On the generation of viscous toroidal eddies in a cylinder. J. Fluid Mech.

95 209-222 
Higdon J. 1985 Stokes flow in arbitrary two-dimensional domains: shear flow over ridges and cavities.

J. Fluid Mech. 159 195-226

C. P. Hills \& H. K. Moffatt 2000 Rotary honing: a variant of the Taylor paint-scraper problem. J. Fluid Mech. (in press)

C. P. Hills 2000 Eddies induced in cylindrical containers by a rotating end-wall. (submitted for publication)

Jeffrey, D. J. \& Sherwood, J. D. 1980 Streamline patterns and eddies in low-Reynolds-number flow. J. Fluid Mech. 96 315-334

S. KHURI 1996 Biorthogonal series solution of Stokes flow problems in sectorial regions. Siam J. Appl. Math. 56, 19-39.

Liron N. \& Shahar R. 1978 Stokes flow due to a Stokeslet in a pipe. J. Fluid Mech. 86 $727-744$

Liu, C. \& Joseph, D. 1977 Stokes flow in wedge-shaped trenches. J. Fluid Mech. 80 443-463

Moffatt, H. K. 1964 Viscous and resistive eddies near a sharp corner. J. Fluid Mech. 18 1-18.

Moffatt H. K. \& Duffy B. R. 1980 Local similarity solutions and their limitations. J. Fluid Mech.

96 299-313.

TAYLOR, G. I. 1962 On scraping viscous fluid from a plane surface. In The Scientific Papers of Sir Geoffrey Ingram Taylor, vol. Iv (ed. G. K. Batchelor), 410-413. Cambridge University Press, 1971. 